\title{
Thermal performance of occupied homes: A dynamic grey-box method accounting for solar gains
}

\author{
Frances P. Hollick*, Virginia Gori, Clifford A. Elwell \\ Physical Characterisation of Buildings Group, UCL Energy Institute, 14 Upper Woburn Place, London WC1H 0NN, UK
}

\section{A R T I C L E I N F O}

\section{Article history:}

Received 14 May 2019

Revised 28 November 2019

Accepted 1 December 2019

Available online 02 December 2019

\section{Keywords:}

Heat transfer coefficient

Heat power loss coefficient

Solar gains

Bayesian statistics

In-situ monitoring

Dynamic grey-box

\begin{abstract}
A B S T R A C T
The accurate determination of the in-use heat transfer coefficient (HTC) of a dwelling can support efficiency improvements and understanding of energy costs, potentially addressing the performance gap. This paper introduces a dynamic grey-box framework combining Bayesian methods and lumped thermal capacitance models for the estimation of the performance of in-use buildings. It focuses on methods to account for solar gains, a significant contributor to the heat transfer. Six simple first-order lumped models of occupied homes are presented, which explicitly include gains from solar radiation with varying complexity. Specifically, the models use solar radiation as a single heat input, divided by façade according to the angle of the sun, and including diffuse radiation. Two case study houses in the UK, monitored over two different seasons, were used to illustrate the models' performance. Bayesian model comparison was used, in conjunction with other methods, to determine the most suitable model for each sub-dataset analysed; this indicates that the most appropriate model is both season and case-study dependent, highlighting the importance of local topography and weather experienced. For each case study, the models selected provided HTC estimates within $15 \%$ of each other, including during the summer, using only 510 days of data. Such techniques have the potential to estimate the thermal performance of dwellings year-round, with minimum disturbance to the occupants and could be developed to improve quality assurance processes for new build and retrofit, identify opportunities for targeted retrofit, and close the performance gap.
\end{abstract}

(C) 2019 The Authors. Published by Elsevier B.V.

This is an open access article under the CC BY license. (http://creativecommons.org/licenses/by/4.0/)

\section{Introduction}

The residential sector accounted for $27 \%$ of global energy consumption in 2010, with emissions from the sector increasing by $14 \%$ in the preceding decade [1]. Reducing carbon emissions associated with the built stock is essential to limit the global temperature increase to $2^{\circ}$; this can be achieved via either fuel switching alone or by also improving the efficiency of the built stock [2]. Although progress is being made in this area, with emissions decreasing in developed countries [1], there is still potential for cost-effective energy demand reduction in the residential sector [2]. However, there is often a significant difference between the designed, or expected, energy use and that observed in reality, termed the performance gap [3-6]. A range of technical and social factors contribute to the performance gap, that affect the design, build, and operation stages of the building [5]. Characterisation of the thermophysical performance of occupied dwellings provides a

\footnotetext{
* Corresponding author.

E-mail address: frances.hollick.15@ucl.ac.uk (F.P. Hollick).
}

mechanism to understand their real energy use; this may be compared to design predictions to identify whether a performance gap is present and subsequently take measures to reduce it. The use of in-situ measurements in this regard is particularly useful as it enables estimates of performance that take into account the impacts of occupant behaviour, installation quality, and degradation of materials on the dwelling performance [5].

The heat transfer coefficient (HTC), often previously and interchangeably referred to as the heat loss coefficient (HLC), is a commonly used and simply understood parameter to describe the thermal performance of a building; it is the heat flow rate per unit temperature difference between inside and outside [7]. There are many proposed methods for determining the HTC of a residential building, which can be separated into static and dynamic approaches. Static methods average the data being analysed over long-enough periods to exclude the impacts of dynamic effects such as thermal mass and thus achieve a quasi-static state. However, dynamic methods aim to characterise how dwellings respond to changes in conditions, including the effect of thermal mass. The method selected to estimate the HTC of a property should address 
the intended purpose of the analysis and the performance insights provided (such as whether the size of the thermal mass or solar aperture are desired); the accuracy and repeatability of methods are also important factors to method selection, in addition to disruptiveness and data requirements.

A key static approach of estimating the HTC is the powertemperature-gradient method, an energy signature method first proposed in [8] and subsequently utilised for a variety of purposes [9-11]. The HTC is simply derived by linear regression as the gradient of the sloped portion of a graph of the total average power consumption against the external temperature. The approach utilises data from occupied dwellings; however, it requires a dataset lasting two seasons at a minimum and assumes that all free heat gains are constant throughout the period analysed [8]. An alternative approach is taken in some related energy signature methods [12-15] by utilising estimates for the magnitudes of the free heat gains over each measurement interval to avoid the assumption they are unchanging. The length of dataset required and implicit steady-state assumptions have prohibited its widespread application, an issue shared by the steady-state methods described in [16] (BEECHAM) and [17] (EBBE).

The coheating test is a static method of HTC characterisation that does not require as lengthy a monitoring period. A constant dwelling internal temperature is maintained through the use of electric heaters for a period of 1 to 3 weeks to minimise thermal mass effects [18]. However, a key disadvantage of this method is that the dwelling must remain unoccupied for the duration of the monitoring, excluding the impact of occupant behaviour on the HTC and consequently potentially making the test prohibitively expensive; additionally, the test is undertaken with planned ventilation sealed and using fans to mix the air. Coheating test results therefore estimate an HTC in a specific dwelling state that does not represent normal operation, and consequently the use of this coheating HTC to represent the total HTC of the dwelling may contribute to a performance gap between real use and test results. Furthermore, the test can only be performed in winter, to ensure that the temperature difference between the internal and external air is sufficiently high to reduce the impacts of measurement error [18]. These necessary conditions to perform a coheating test make it difficult to integrate into the construction process: only houses available in winter may be tested and a minimum two-week period between completion and handover is required. These issues are exacerbated for retrofitted homes, where the dwelling may not be unoccupied for any period of time.

An extension to the coheating test to allow it to take place in summer is presented in [19], where the heating power from solar gains is added to that measured from the electrical heaters. This heating power is calculated based on typical transmittance values, and the solar radiation is either directly measured on each façade, which may not always be practical in a measurement campaign, or the global horizontal data are translated. The method gives HTC estimates with accuracies of $\pm 15 \%$ [19].

Dynamic methods aim to characterise, rather than average out, effects such as thermal mass and can allow a much shorter monitoring period to determine the HTC of a dwelling. The methods described in [20-22] utilise variations of the coheating test with free running periods to estimate the thermal mass using the cooling curve. However, despite this extension, these methods share the limitations of the coheating test that the property must be unoccupied, often in a different state to that in-use, and ideally the test takes place during winter.

Many proposed dynamic methods use the assumption of lumped matter, where a system is modelled as a series of thermal masses ('lumps'), within which the temperature is homogeneous, joined by thermal resistances [23, p. 260-261]. Models of this form may be customised to represent property characteristics that may be readily physically interpreted. The simplest of these models include a single thermal resistance and capacitance and estimate the product of these two parameters, the thermal time constant. One such method is presented in [24]; however, this simple approach does not account for occupancy or other heat inputs.

A more complex model for whole building representation (or, as in this case, simulation) is outlined in [25], where the dwelling is represented by three thermal capacitances and five resistances representing ventilation and fabric heat losses. A similar approach is also utilised in [26], where two adjoined test rooms are represented by a system of four thermal capacitances, one for both the floor and air in each zone. Heat inputs from solar radiation and the heating system are included; the former as a single heat input split between the floor and air capacitances with the assumption that $60 \%$ of the glazing area is the solar aperture. The solar aperture of a dwelling is dependent on both the amount of glazing, the orientations of said glazing and any shading of the glazing. The latter is non-constant, as it is affected by the time of year and occupant behaviour (for example, curtain usage) and thus assuming this value could potentially lead to errors in the modelled heat input from solar radiation.

An alternative to [26]'s method of accounting for solar gains has been proposed to predict future thermal loads of buildings, rather than thermal performance parameters [27-29]. The highly complex models require significant input data or assumptions about the construction performance. Solar gains are addressed by estimating the sol-air temperature based on the building location and the forecast maximum external temperature, and cloud cover. Utilising the sol-air temperature as opposed to solar radiation measurement introduces additional complexity and assumptions.

Characterisation of the thermal performance of occupied dwellings could be used to support the implementation of policies and the improvement of practices to reduce energy demand. The method requires an accuracy suitable for identifying any notable performance gap, and should cause minimal disruption to occupants. However, at present there is no widely accepted way to estimate the HTC of an occupied dwelling, particularly outside the winter months, and accounting for solar gains. This paper presents and compares a series of methods of addressing solar gains in lumped thermal capacitance models of buildings using easily available weather data and minimal assumptions; the analysis uses Bayesian techniques for thermophysical parameter estimation. Solar radiation data is included in the models as a heat gain to the internal thermal mass, multiplied by an effective solar aperture. The effective solar aperture is an estimated parameter, encompassing the glazing fraction and shading as well as other factors including the reflectivity of the glass and window opening. These effects can either be averaged over the entire dwelling or characterised for each façade separately. The latter is preferable as it better reflects the dynamic nature of these effects. Models including the impact of diffuse radiation are also presented. Bayesian model comparison is used to objectively identify the model that best describes the data. The results from the different models, and their physical interpretation, are discussed.

In the following section the lumped thermal models developed in this research are described, along with details of the data used and the analysis performed. In Section 3 the results of applying these models are discussed, and the most appropriate model for each data period is identified; the conclusions of the paper are presented in Section 4.

\section{Methods}

The grey-box models developed to estimate the heat transfer and heat power loss (HPLC) coefficient of occupied dwellings are discussed in this section. Firstly, the models are described, fol- 


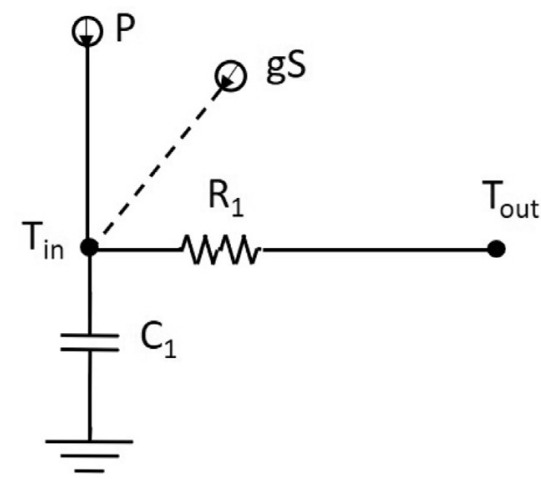

Fig. 1. Equivalent electrical circuits of the 1C1R1P (solid lines) and 1C1R1P1S (solid and dashed lines) models, where the model names reflect the number of thermal masses $(C)$, thermal resistances $(R)$, power inputs $(P)$ and solar inputs $(S)$. The house is represented as a single effective thermal mass, $C_{1}$, at the area-weighted average internal temperature, $T_{\text {in }}$. It has a thermal resistance, $R_{1}$, to the external temperature, $T_{\text {out }}$, and an effective solar aperture, $g$.

lowed by an overview of Bayesian statistical analysis. The data used in this study is outlined, and finally the details of the analysis setup are provided.

\subsection{Theory and calculations}

The analysis presented in this paper represents whole building heat loss through highly simplified models, utilising the lumped matter discipline followed by Bayesian inference. The method is similar to that developed to estimate the thermal performance of building elements, developed by [30], which employs the Occam's razor approach of Bayesian model comparison to enable the most appropriate models to be selected.

\subsubsection{Lumped thermal capacitance models}

The lumped models of heat transfer in an occupied house are presented below, incorporating increasingly complex methods to account for solar gains: from a model with no explicit representation of solar gains, through models with a single solar gain input, and a model that represents the gain on each façade of the building, to finally a model that incorporates the gains at different façades in addition to diffuse radiation. These models produce estimates for the thermal resistance between the internal and external temperatures, the inverse of which is the HTC or HPLC. The differences between these two parameters are discussed further in Section 2.2.2.

a) Single thermal mass model with power input (1C1R1P) The base model of the house (1C1R1P, shown in Fig. 1) takes no explicit account of the solar gains. It comprises a single effective thermal mass with units $\mathrm{JK}^{-1}, C_{1}$, with a thermal resistance with units $\mathrm{KW}^{-1}$ to the external temperature, $R_{1}$, and a heat gain in units of $\mathrm{W}$ equal to the combined electricity and gas consumption multiplied by an efficiency $\lambda$, giving $P(t)=\lambda P_{\text {gas }}(t)+P_{\text {elec }}(t)$. The use of $\lambda$ is discussed further in Section 2.2.2. The resultant differential equation is:

$C_{1} \frac{d T_{\text {in }}(t)}{d t}=\frac{T_{\text {out }}(t)-T_{\text {in }}(t)}{R_{1}}+P(t)$,

where $T_{\text {out }}(t)$ and $T_{\text {in }}(t)$ are respectively the external and internal air temperatures at time $t$, with the latter assumed to be the temperature of the effective thermal mass. The inputs to this model are $T_{\text {out }}(t)$ and $P(t)$, with the data for $T_{\text {in }}(t)$ used for optimisation and the remaining quantities $\left(C_{1}, R_{1}\right)$ in Eq. (1) the parameters to be estimated.

b) Single thermal mass model with power and solar input (1C1R1P1S) The second model presented here extends the 1C1R1P

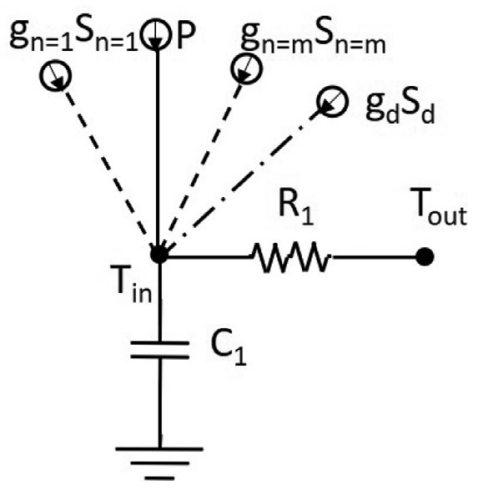

Fig. 2. Equivalent electrical circuit representation of the 1C1R1PmS (solid and dashed lines), and 1C1R1PmSD (solid, dashed, mixed lines).

model by incorporating solar gains as a single term across the entire building (a single, average, solar aperture): the 1C1R1P1S model (Fig. 1). The data inputs are the same as for the previous model, with the addition of the solar radiation, $S$. The solar aperture, $g$, has units of $\mathrm{m}^{2}$, and represents the area of the building's external surfaces that transfers heat from solar radiation to the internal thermal mass; this is similar in physical form to that in [26]. As a differential equation:

$C_{1} \frac{d T_{\text {in }}(t)}{d t}=\frac{T_{\text {out }}(t)-T_{\text {in }}(t)}{R_{1}}+P(t)+g S(t)$.

c) Single thermal mass model with power and separate solar input per façade (1C1R1PmS) The third model presented here attempts to capture the complexity of orientation, glazing size and position, and shading, through incorporating a solar aperture for each façade of the building. Taking $m$ as the number of non-North facing façades, the analysis can be performed in the range $1 \leq$ façades considered $\leq m$. This 1C1R1PmS model (Fig. 2) utilises the same input data as the 1C1R1P1S model above, with solar input for each façade adjusted by the difference between the orientation of the wall and the azimuth angle of the sun, $z$, at a given time. It is in all other respects identical to the 1C1R1P1S model. This is governed by the following:

$C_{1} \frac{d T_{\text {in }}(t)}{d t}=\frac{T_{\text {out }}(t)-T_{\text {in }}(t)}{R_{1}}+P(t)+\sum_{n=1}^{n=m} g_{n} S_{n}(t)$

where

$S_{n}(t)=\left\{\begin{array}{l}S(t) \cos (z(t)-0) \\ 0\end{array} \quad\right.$ for $|z(t)-o| \leqslant \frac{\pi}{2}$

where $o$ is the orientation of façade $n$ with respect to South in units of radians. Up to four mutually perpendicular orientations may be included; whilst more complex buildings with more façades can be modelled in this way, the apertures estimated would not then be comparable to those, for instance, estimated in a coheating test. An example of solar radiation separated in this manner is shown in Fig. 3, for case study house A (described in Section 2.2.1).

d) Single thermal mass model with power, direct solar input per façade and diffuse solar input (1C1R1PmSD) The 1C1R1PmS model accounts only for direct solar radiation, and does not support any heat input to a North-facing façade. This is addressed through the final model presented here: the 1C1R1PmSD model, shown in Fig. 2, where an additional solar input is added at all times representing the diffuse solar radiation, $S_{\mathrm{d}}$. This model uses the same input data as the previous two presented. It is described by:

$C_{1} \frac{d T_{\text {in }}(t)}{d t}=\frac{T_{\text {out }}(t)-T_{\text {in }}(t)}{R_{1}}+P(t)+\sum_{n=1}^{n=m} g_{n} S_{n}(t)+g_{d} S_{\mathrm{d}}(t)$, 


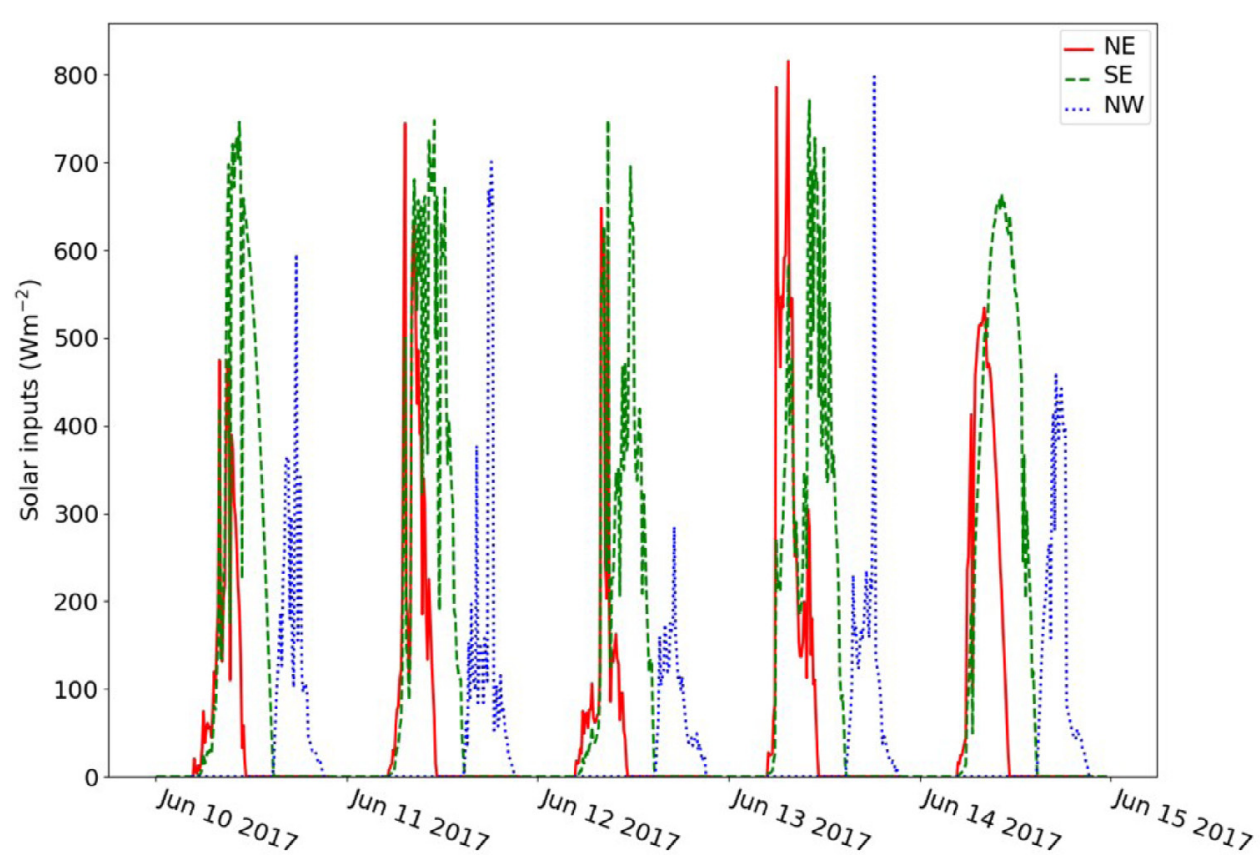

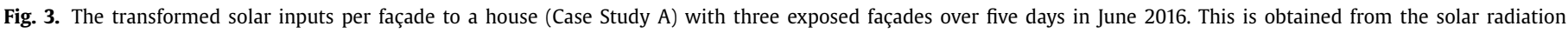
data using Eq. (4).

where $S_{\mathrm{d}}(t)$ is the diffuse solar radiation calculated from an empirical relationship between the diffuse and total solar radiation presented in [31]:

$S_{\mathrm{d}}(t)=S(t) \begin{cases}1.0-0.249 k_{\mathrm{T}}(t) & k_{\mathrm{T}}(t)<0.35 \\ 1.557-1.84 k_{\mathrm{T}}(t) & 0.35 \leqslant k_{\mathrm{T}}(t) \leqslant 0.75 \\ 0.177 & k_{\mathrm{T}}(t)>0.75\end{cases}$

The clearness index, $k_{\mathrm{T}}$, is the ratio of the total radiation at the surface of the Earth, $S$, to the extraterrestrial radiation, $S_{0}$. The latter is defined here by the following equation, taken from Duffie and Beckman [32, p. 37]:

$S_{0}=S_{\mathrm{SC}}\left(1+0.033 \cos \left(\frac{2 \pi i}{365}\right)\right) \cos (z)$

where $i$ represents the day number of the year and $S_{\mathrm{SC}}$ is the solar constant, $1367 \mathrm{Wm}^{-2}$ [32]. The solar inputs for each façade are then calculated as for the 1C1R1PmS model, after the calculated diffuse radiation is subtracted from the measured radiation:

$S_{n}(t)=\left\{\begin{array}{l}\left(S(t)-S_{\mathrm{d}}(t)\right) \cos (z(t)-0) \\ 0\end{array}\right.$

for $|z(t)-o| \leqslant \frac{\pi}{2}$ and $(S(t)$

The models outlined in this section are evaluated with respect to the data using the Bayesian techniques described in the following section to produce estimates for the model parameters. The global structural identifiability of the models was assessed utilising the transfer function approach [33], with all found to meet this requirement under the relevant datastreams combination used in this paper (i.e. dynamic non-zero temperature timeseries, and non-zero power and solar radiation observations) as shown in the Appendix.

\subsubsection{Bayesian inference with MCMC sampling}

The parameters of the models outlined in Section 2.1.1 above are evaluated using Bayesian inference with Markov Chain Monte Carlo (MCMC) sampling. Bayes' theorem gives:

$p(\theta \mid y, H)=\frac{p(y \mid \theta, H) p(\theta \mid H)}{p(y \mid H)}$, where $p(\theta \mid y, H)$ is the joint posterior probability distribution of the vector of parameters $\theta$ given the data $y$ and model $H ; p(y \mid \theta, H)$ is the likelihood function, describing the ability of the model to explain the data; $p(\theta \mid H)$ is the prior probability distribution of $\theta$; and finally $p(y \mid H)$ is the marginal distribution of the data, which acts as a normalising constant [34].

The posterior is often mathematically intractable for complex models, therefore MCMC sampling is used to simulate the posterior parameter distribution, by drawing samples iteratively from it and ensuring that with each step the process produces a proposal posterior distribution that is at least as close as the current distribution to the target. An affine-invariant ensemble sampler is used in this work, which, among other advantages, has a shorter autocorrelation time than traditional MCMC samplers and is thus a more efficient sampler [35,36].

The implementation of Bayesian inference with MCMC sampling results in an estimate of the probability distribution of the model solution, which can be marginalised to that for each individual parameter and illustrate correlation between parameters. This inforfadtibh $\tau a$ a be used to explore potential issues around the estimated parameter space, such as the identification of significant covariance and possible practical identifiability issues [37], truncation of distributions and any multi-modal behaviour, supporting greater investigation into the validity of the solution than a single maximum a posteriori (MAP) estimate. The relationships between variables have also been shown to support the physical interpretation of solutions, providing greater insight into in-situ performance than is possible with single estimates of parameters [38].

A key advantage of a Bayesian framework is its model comparison method; comparison of the evidences (i.e. the marginal distributions of the data) embodies the principle of Occam's razor in that more complex models are penalised, unless the improvement in the fit of the parameters to the data justifies the additional complexity [39]. The evidence for a model is estimated using Eq. (9) as described in [30] and thus penalises models with increased prior space resulting from higher numbers of parameters $[34,40]$. 


\subsection{In-situ monitoring and analysis}

In this section the datasets used in this paper are described, followed by the specific details of the application of the analysis methods introduced above. Two case studies are presented; one semi-detached, occupied house with unusual water heating patterns (discussed below), and one detached house which was unoccupied but maintained a space heating cycle and occasional electricity use.

\subsubsection{Case studies}

The first dwelling (hereafter referred to as Case Study A [41]) is a semi-detached, two-storey, occupied house near Letchworth Garden City (UK), with solid wall construction, solar photovoltaic panels and a system boiler with a stated efficiency of $88.8 \%$. Its external façades have NE, SE and NW orientations. A coheating test performed in 2016 gave an HTC of $141 \mathrm{WK}^{-1}$ [42].

An extensive monitoring campaign was conducted on Case Study A, recording a wide range of parameters to characterise the thermal performance, whilst balancing this against the need to limit the intrusiveness and disruption to occupants. The air temperature in every room was measured using Eltek GD10 temperature and relative humidity transmitters [43]; the $\mathrm{CO}_{2}$ concentration in the main downstairs room with an Eltek $\mathrm{GW} 47 \mathrm{CO}_{2}$ transmitter [44], and the electricity and gas consumption with Eltek GC62 pulse transmitters [45]. These parameters were sampled and recorded at $10 \mathrm{~min}$ intervals, with a weather station approximately 10 miles away recording the external air temperature and global solar radiation at the same intervals using an Eltek OD14J external temperature transmitter [46] and a Kipp \& Zonen CMP3 pyranometer [47] respectively. Ideally the weather station would have been sited closer to the property, but this was not possible due to conservation and aesthetic concerns regarding the site. On-site external temperature was measured for a short period, and agreed closely with that from the weather station. The total electricity use was calculated at each time interval as the sum of the import from the grid and the generation from the photovoltaic panels, minus the export to the grid.

The second property (hereafter Case Study B) is a detached house over three floors with a winter garden (SE facing) and skylights, in addition to standard glazing on two façades (NW and SE), near York, UK. It has partially insulated cavity walls and a timber frame construction, with a combination boiler with a stated efficiency of $91.2 \%$. A coheating test was performed giving an HTC of $133 \mathrm{WK}^{-1}$ [48]. The house was unoccupied during the period when the data used in this paper was collected; however, a daily gas space heating schedule was in operation throughout, with occasional electricity use due to its purpose as a show home. These sources of energy use in the dwelling approximate an occupied state, but do not represent patterns of water heating, door and window opening, and other occupant-driven energy uses. The total electricity use at each time step was calculated as the total of the sub-metered electricity. The air temperature in the monitored rooms and the electricity and gas use was recorded at 10 min intervals, with the external air temperature and solar radiation recorded at a nearby location, again every $10 \mathrm{~min}$. Excluding the gas data, the above were recorded using a system of Eltek transmitters and dataloggers; the gas data was collected using a Revenco COPMV2 pulse transmitter [49].

\subsubsection{Gas consumption and boiler efficiency}

The calculation of the HTC requires measurement of the heat delivered to the property, or its estimation from related data. Heat delivered may be measured using heat meters, neglecting case losses of the heating plant if it is located within the thermal envelope of the dwelling, as here. For a boiler, as fitted to the case studies investigated, such metering requires the pipework near the boiler to be revised to accommodate heat meters on the cold inlet, hot water, flow and return, incurring significant costs and disruption. These issues prevented the installation of heat meters during this research.

The nominal efficiency of the heating plant multiplied by the gas use provides an estimate of the heat delivered to the property, neglecting adjustments to account for use of gas through appliances other than the boiler, such as gas fires and cookers. These adjustments are likely to be unnecessary for Case Study B, as it was not occupied. Such an estimate neglects gains from case losses of the boiler into the dwelling and assumes that the boiler operates at a known efficiency throughout the year. Methods exist to estimate the seasonal boiler efficiency, such as those employed in the UK's National Calculation Method, the Standard Assessment Procedure (SAP) [50], addressing the relative demands of water and space heating over the year, plus changes to the efficiency of water heating. However, they do not account for variations in-situ boiler performance due to factors such as the mismatch of heat demand to boiler output, and consequent on-off cycling behaviour, which can have a significant impact on boiler efficiency [51-53].

Alternatively to estimating the efficiency of the heating plant, the combined whole dwelling fabric and heating system performance may be estimated by using the gas delivered to the property as the energy input. This approach produces an estimate of the heat power loss coefficient, HPLC, as defined by [54]. Whilst not directly comparable to the HTC, the HPLC avoids assumptions of the efficiency of the heating plant and may be readily related to occupants' bills and carbon reduction targets. Both the HTC derived using a constant boiler efficiency, $\lambda$, and the $\operatorname{HPLC}(\lambda=1)$ (see Section 2.1.1) are calculated as the inverse of the estimated thermal resistance, $R_{1}$, and reported in this paper to provide insight into the difference between these two measures.

\subsubsection{Analysis}

This section provides the details of the analysis described in Section 2.1, applied to the data described in Section 2.2.1. The solar radiation data was transformed to that received by a vertical surface utilising the diffuse fraction, calculated as above, and the altitude of the Sun at any given time. In the transform of the measured solar radiation data the transformed radiation was limited to not exceed the total extraterrestrial radiation at that time; this prevented possible numerical issues introduced, for example, by reflected radiation on the pyranometer at low and negative solar altitudes or measurement noise.

The differential equations described in Section 2.1.1 were approximated using the bilinear transform, as shown in [39], and solved for $T_{\text {in }}$, the area-weighted average internal temperature for each property, which is assumed to be the temperature of the internal thermal mass. A Gaussian likelihood was used to assess the discrepancy between the time series of $T_{\text {in }}$ predicted by the model and the measured one. The residuals were assumed independent and identically distributed. During this optimisation stage all parameters are rescaled according to their prior limits to ensure they are of comparable magnitudes.

The priors used for the model parameters (e.g., $R_{1}, C_{1}, g, T_{\text {in }}$ ) were uniform with large ranges, as shown in Table 1 . These ranges were selected to assign equal probability to all physically possible values of the parameters, for example avoiding a negative effective solar aperture, whilst not making assumptions about the performance of the dwelling, or, in the absence of evidence, the likely distribution of dwelling thermal performance characteristics. To ensure that the priors selected were not over-informative and that the likelihood contributed sufficiently to the final estimates, it was checked that the posterior standard deviations were less than 
Table 1

The ranges of the uniform prior distributions for all models.

\begin{tabular}{llll}
\hline Parameter type & Unit & Minimum value & Maximum value \\
\hline Thermal resistance, $R_{1}$ & $\mathrm{KW}^{-1}$ & $1.0 \times 10^{-4}$ & 1.0 \\
Thermal mass, $C_{1}$ & $\mathrm{JK}^{-1}$ & 0.1 & $5.0 \times 10^{8}$ \\
Temperature, $T_{\text {in }}$ & $\circ$ & -5.0 & 40.0 \\
Solar aperture, $g$ & $\mathrm{~m}^{2}$ & $1.0 \times 10^{-7}$ & $1.0 \times 10^{3}$ \\
\hline
\end{tabular}

$10 \%$ of those of the priors [55]. Priors were kept constant for each model to enable model comparison using the evidences.

These choices of prior and likelihood, substituted into Eq. (9), produce the following unnormalised posterior:

$$
\begin{aligned}
& p(\theta \mid y, H) p(y \mid H)= \\
& \quad(2 \pi)^{-\frac{n}{2}}\left|\sigma^{2} I_{n}\right|^{-\frac{1}{2}} \exp \left(-\frac{1}{2} r(\theta)^{T}\left(\sigma^{2} I_{n}\right)^{-1} r(\theta)\right) \prod_{j} \frac{\mathbf{1}_{\left[p_{j}, q_{j}\right]}\left(\theta_{j}\right)}{q_{j}-p_{j}}
\end{aligned}
$$

where $n$ is the number of observations in the datastream analysed (i.e. internal temperature in this case, $\left.T_{\mathrm{in}, \mathrm{m}}\right) ; r(\theta)=T_{\text {in, } \mathrm{e}}(\theta)-T_{\mathrm{in}, \mathrm{m}}$ is the vector of residuals between the time series of the internal temperature estimated from a lumped thermal capacitance model (defined in Section 2.1.1) and the measured one; $\sigma^{2}$ is the variance of the distribution of the residuals; $I_{n}$ is an identity matrix of dimension $n$; $\theta_{j}$ denotes each parameter (e.g., $R_{1}, C_{1}, T_{\text {in }}, g$ ) in the vector of parameters $\theta ; p_{j}$ and $q_{j}$ are the lower and upper limits of the prior for each parameter; and finally $\mathbf{1}_{\left[p_{j}, q_{j}\right]}\left(\theta_{j}\right)$ is the indicator function, which is one when $\theta_{j} \in\left[p_{j}, q_{j}\right]$ and zero otherwise.

As a preliminary step, the SciPy Basinhopping function [56] was used to maximise the function in Eq. (10) and obtain the MAP estimates of the parameters. The information matrix of the parameters was computed as the Hessian of the minus natural logarithm of Eq. (10) at the MAP. Its condition number was calculated to investigate the structural local identifiability of the model [37]. Conversely, the practical identifiability was assessed through plots of the marginalised joint distribution of pairs of parameters obtained from the MCMC chains as follows.

To obtain the posterior distributions of the parameters in addition to a point estimate (the MAP), MCMC sampling was performed using the EMCEE Python module [35], with the walkers initialised using an overdisperse distribution centred at the MAP [40]. The convergence of the posterior parameter distributions was assessed through examination of the corresponding MCMC traces and integrated autocorrelation times. The burn in was removed after plotting the MCMC traces, but before plotting the posterior distributions and their covariances. For all analyses presented here 100 MCMC walkers were used for 2000 iterations initially, then the iterations were increased as necessary [34]. A burn in of 250 iterations was found to be suitable for all cases [34]. The final HTC or HPLC estimate was, for all models, calculated as the inverse of $R_{1}$, the thermal resistance between the internal and external temperatures.

For Case Study A it was found that five days of data was sufficient for estimates of the HTC (and HPLC) to converge, whilst for Case Study B ten days of data were required. As Case Study B is a far more complex building, this is not unexpected. In the absence of a standardised method to determine the optimal length of time series to be analysed for whole-building thermophysical characterisation, this was determined with an approach based on that in [57] for a static method of estimating wall U-values. The 1C1R1P and 1C1R1PmS models were applied to increasing numbers of complete days of data until the resulting HTC was within $10 \%$ of that obtained $24 \mathrm{~h}$ previously. This criterion is less strict than the 5\% used in [57], due to the increased complexity of an occupied house in comparison to a single element. For both case studies, data from within and outside the winter heating season was selected for analysis; for A this was from November 2016 and the following June, and for B from February and April of 2012. The HTC stabilisation was explored on all selected data, with the longest required dataset chosen for all seasons in each case study. There was no hot water production for Case Study B and the June 2017 data for Case Study A, whereas for November 2016 in Case Study A there was constant water heating with no evidence of use in the return temperatures. It is advantageous to analyse as short a period of data as possible, as the models used assume that all thermophysical properties are constant. Analysing multiple short data periods from a larger dataset therefore facilitates the study of parameter changes due to changes in ventilation, shading, and occupant behaviour [30]. The ability to utilise short measuring periods also enables the method to be used for standalone monitoring campaigns of limited duration.

To enable comparisons between models and assess which best represents the data, the root mean square error (RMSE) of the predictions for $T_{\text {in }}$ and the evidence for each application of a model were computed.

\section{Results and discussion}

In this section the results of all models described in Section 2.1.1, for both case studies, are presented and compared to one another and to the coheating test results. The most effective model for each case study is also identified and discussed, with the numeric parameter estimations given the means of their marginalised posterior probability distributions.

\subsection{Model evaluation and selection}

The appropriateness of a model for the data analysed is initially assessed through examination of plots of the MCMC traces, posterior parameter distributions, and their covariances. This facilitates the identification of problems and challenges, such as multimodal parameter distributions and the use of insufficiently broad prior distributions leading to truncation of parameter distributions. The evidence for each of the models (see Eq. (9)) is also compared, to give an objective comparison of model fit that takes into account the increased goodness of fit that is typically observed as the number of parameters is increased. Finally, the RMSE for the predictions of the average internal temperature $\left(T_{\text {in }}\right.$, the temperature of the internal thermal mass) is considered. This section focuses on discussion of the 1C1R1P1S, 1C1R1PmS and 1C1R1PmSD models, which were found to represent the observed data significantly better than the simpler 1C1R1P model. The separated solar models (1C1R1PmS and 1C1R1PmSD) were applied to the data for Case Study A. Assessment of the MCMC traces and posterior distributions of the results from the $1 \mathrm{C} 1 \mathrm{R} 1 \mathrm{PmS}$ model for the June data from Case Study A indicated that not all façades were significant, as shown in Fig. 4a. These images are from the analysis producing the HPLC estimate, however the same patterns were observed for the analysis resulting in the HTC. The posterior distribution for $g_{3}$, the effective solar aperture of the NW façade (Fig. 4b), shows that the value is likely very close to the lower boundary of the prior $\left(\sim 0 \mathrm{~m}^{2}\right)$, with the peak at approximately zero. These results suggest that the third solar aperture $g_{3}$ (NW façade) is unnecessary, as confirmed by comparison of the evidence which was smaller when $g_{3}$ was included than without it. This façade (and consequently the $g_{3}$ parameter) was therefore excluded and the $1 \mathrm{C} 1 \mathrm{R} 1 \mathrm{P}(\mathrm{m}-1) \mathrm{S}$ model was applied. The corresponding covariances and distributions are shown in Fig. 4b), where all the traces have converged and the posterior parameter distributions are approximately Gaussian in appearance. 


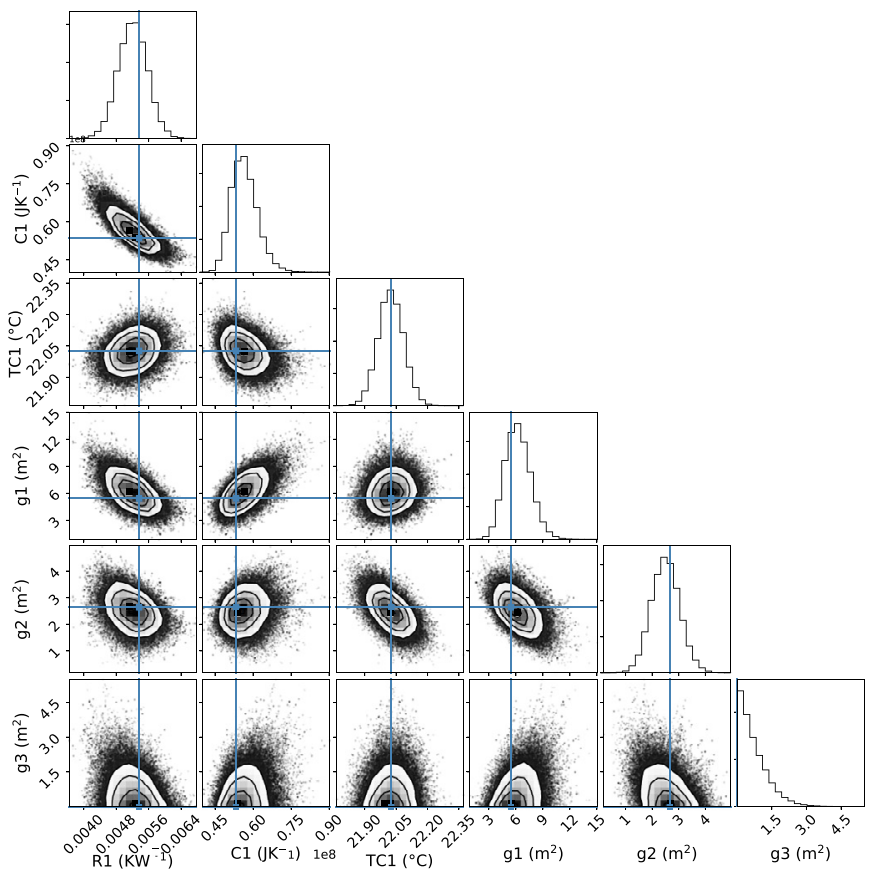

(a)

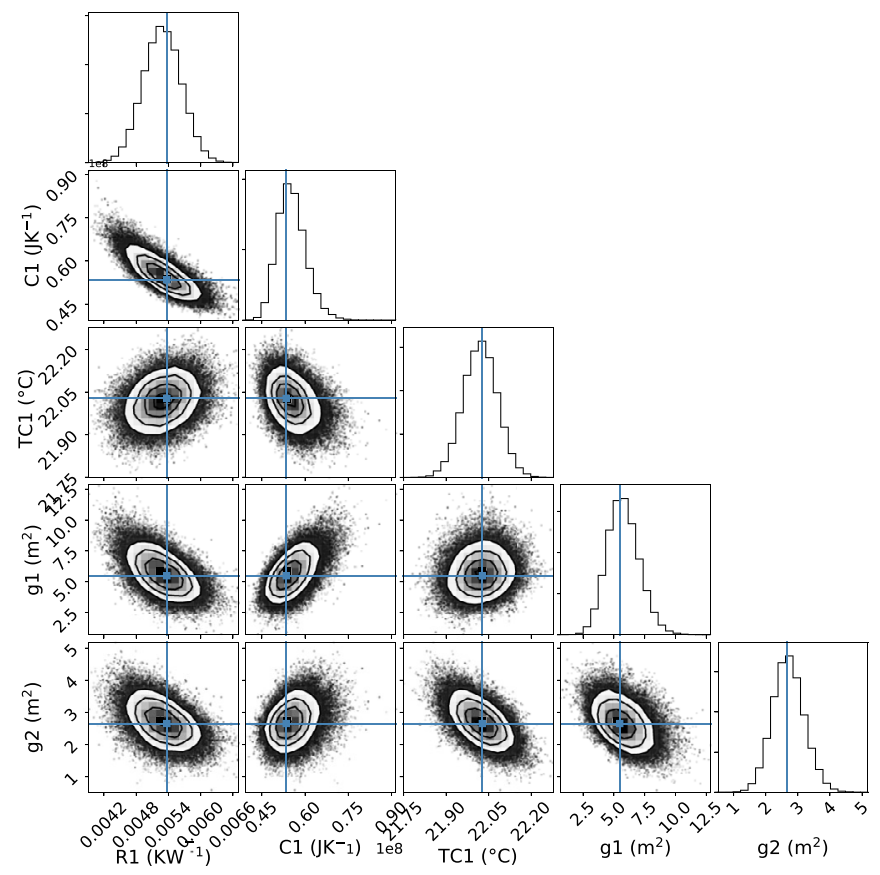

(b)

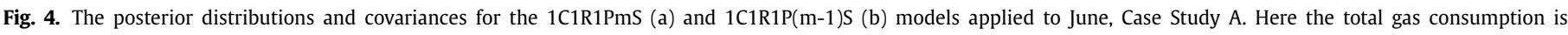

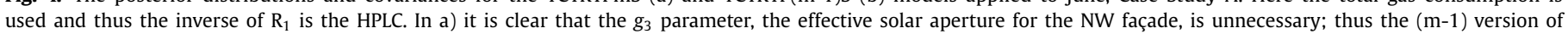
the model was applied. The approximately Gaussian posterior distributions shown in c) demonstrate this further.

Table 2

The results for the selected models for the two time periods from Case Study A; $1 \mathrm{C} 1 \mathrm{R} 1 \mathrm{P}(\mathrm{m}-1) \mathrm{S}$ for June, and $1 \mathrm{C} 1 \mathrm{R} 1 \mathrm{P}(\mathrm{m}-1) \mathrm{SD}$ for November. $g_{2}$ is estimated for June, and $g_{3}$ for November.

\begin{tabular}{llllll}
\hline & $\mathrm{R}_{1}\left(\mathrm{mKW}^{-1}\right)$ & $\mathrm{C}_{1}\left(\mathrm{MJK}^{-1}\right)$ & $\mathrm{g}_{1}\left(\mathrm{~m}^{2}\right)$ & $\mathrm{g}_{2 / 3}\left(\mathrm{~m}^{2}\right)$ & $\mathrm{g}_{\mathrm{d}}\left(\mathrm{m}^{2}\right)$ \\
\hline Jun, HTC & $5.9 \pm 0.4$ & $50 \pm 4$ & $5 \pm 1$ & $2.4 \pm 0.5$ & - \\
Jun, HPLC & $5.3 \pm 0.4$ & $55 \pm 5$ & $6 \pm 1$ & $2.7 \pm 0.5$ & - \\
Nov, HTC & $6.9 \pm 0.3$ & $50 \pm 3$ & $50 \pm 20$ & $30 \pm 7$ & $6 \pm 1$ \\
Nov, HPLC & $6.1 \pm 0.2$ & $50 \pm 3$ & $50 \pm 30$ & $26 \pm 6$ & $5.7 \pm 1.0$ \\
\hline
\end{tabular}

The temperature predictions for the $1 \mathrm{C} 1 \mathrm{R} 1 \mathrm{P}(\mathrm{m}-1) \mathrm{S}$ model for Case Study A June data are shown in Fig. 5, plotted alongside the area-weighted average internal temperature of the dwelling. The NW façade was excluded from the analysis. The fit of the modelled to the measured data appears good, with a low RMSE value of $0.12 \mathrm{WK}^{-1}$ for both plots. The residuals, whilst not consistently biased, do not appear uncorrelated indicating that as expected this simplified model omits some heat transfer processes present in the building. The parameter estimates from this model are shown in Table 2.

The HPLC estimated for these data by the $1 \mathrm{C} 1 \mathrm{R} 1 \mathrm{P}(\mathrm{m}-1) \mathrm{S}$ model is $(190 \pm 10) \mathrm{WK}^{-1}$ and the HTC is $(170 \pm 10) \mathrm{WK}^{-1}$, as given in Table 4. The HTC, where the nominal boiler efficiency has been applied to the gas consumption data, is lower than the HPLC as expected.

The temperature predictions shown in Fig. 6 are those produced by the 1C1R1P(m-1)SD model for November in Case Study A, having the greatest evidence for these data. In contrast to Fig. 5, the resultant temperature predictions plotted alongside the areaweighted average internal temperature in Fig. 6 do not follow the trends of the data as closely, which is reflected in the increased RMSE values. A key difference in heat flows between the November and June data is the differing operation of the boiler, with no water heating in June, but constantly high flow and return temperatures to the hot water cylinder in November. The gas use for this water heating is captured in this model as a direct heat gain to the dwelling; however, the real behaviour is clearly far more complex, with gas use for water heating decoupled from the direct gains by its storage in the hot water tank, and by losses through waste water, resulting in a less accurate temperature prediction. The solar aperture values are large for data from November and there is significant error in the parameter estimates; this illustrates the failure of the model to clearly capture the internal temperature patterns within the dwelling, potentially a consequence of the complex heat gains and losses from the water heating.

The estimated HPLC for the November data for Case Study A is $(163 \pm 5) \mathrm{WK}^{-1}$, and the HTC is $(145 \pm 6) \mathrm{WK}^{-1}$. Unlike the model with the highest evidence for this Case Study house for the June data, the diffuse radiation is included in this model and may reflect the increased proportion of cloud cover in November. The orientation of the façades included in the models with the highest evidence is also different in June and November; the NE façade is not included in November, being replaced with the NW façade, while the SE facing façade remains. Such differences may be expected for dwellings surrounded by complex topography, where seasonal differences in shading are likely.

The model with the highest evidence for the February data from Case Study B is also the $1 \mathrm{C} 1 \mathrm{R} 1 \mathrm{P}(\mathrm{m}-1) \mathrm{SD}$ model, excluding the NW façade. The resultant temperature predictions are shown alongside the area-weighted average internal temperature data in Fig. 7, and the parameter estimates are found in Table 3. This model produced a HPLC estimate of $(137.4 \pm 0.9) \mathrm{WK}^{-1}$ and a HTC of $(125.6 \pm 0.9) \mathrm{WK}^{-1}$.

The model without separate consideration of diffuse radiation $(1 \mathrm{C} 1 \mathrm{R} 1 \mathrm{P}(\mathrm{m}-1) \mathrm{S})$ had the largest evidence for the April data from Case Study B, with the same façade (NW) excluded as for February. The temperature predictions resulting from this analysis are shown 


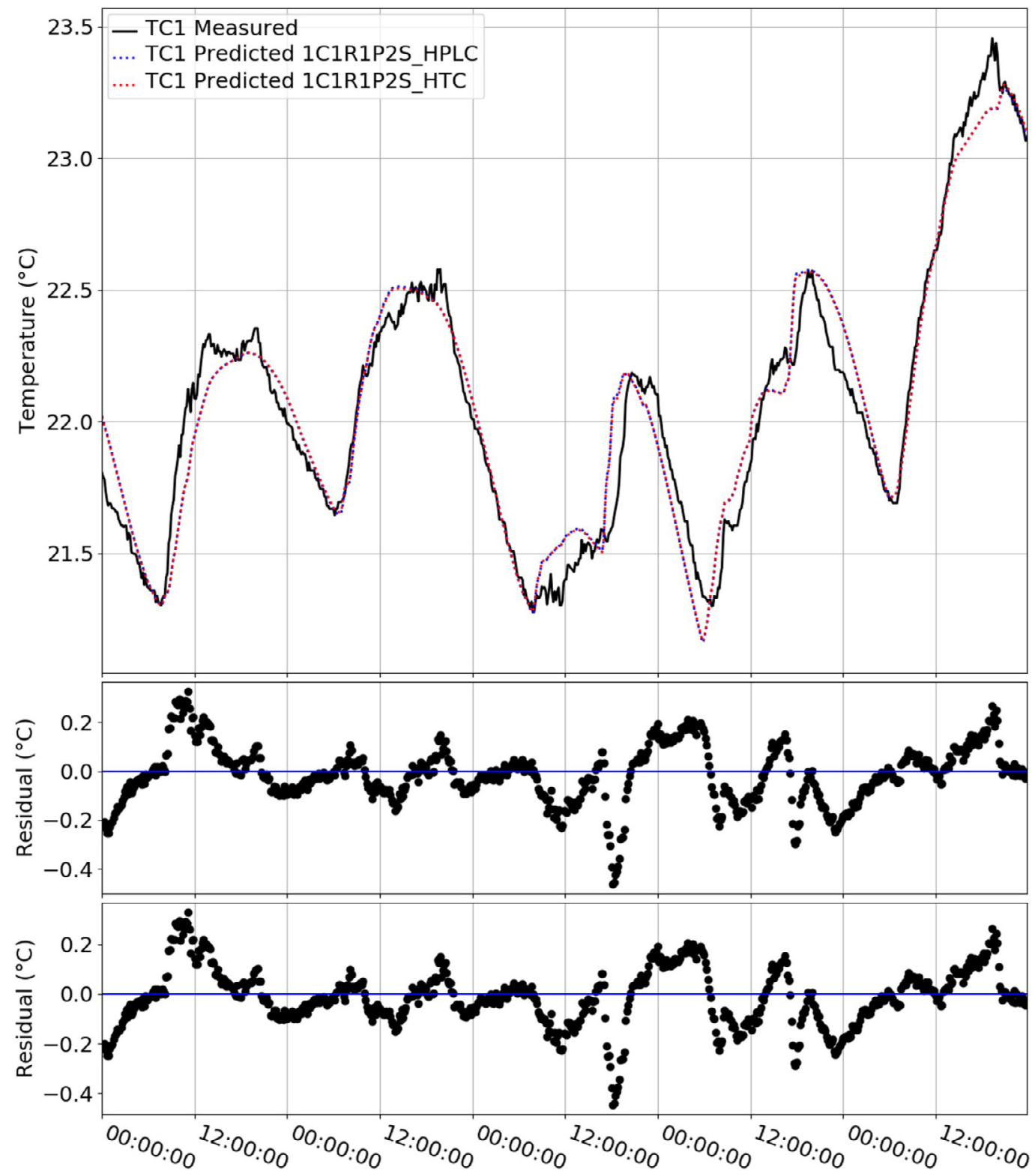

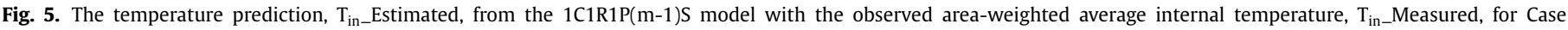
Study A over 5 days in June 2017. The residuals are in the order presented in the legend; this is the case for all following figures also.

Table 3

The results for the selected models for the two time periods from Case Study B; 1C1R1P(m-1)S for April and 1C1R1P(m-1)SD for February.

\begin{tabular}{lllll}
\hline & $\mathrm{R}_{1}\left(\mathrm{mKW}^{-1}\right)$ & $\mathrm{C}_{1}\left(\mathrm{MJK}^{-1}\right)$ & $\mathrm{g}_{1}\left(\mathrm{~m}^{2}\right)$ & $\mathrm{g}_{\mathrm{d}}\left(\mathrm{m}^{2}\right)$ \\
\hline Apr, HTC & $6.56 \pm 0.06$ & $55 \pm 1$ & $16.8 \pm 0.5$ & - \\
Apr, HPLC & $6.03 \pm 0.05$ & $61 \pm 1$ & $18.4 \pm 0.57$ & - \\
Feb, HTC & $7.96 \pm 0.05$ & $47.6 \pm 0.7$ & $9.5 \pm 0.2$ & $10.1 \pm 0.3$ \\
Feb, HPLC & $7.28 \pm 0.05$ & $51.8 \pm 0.8$ & $10.6 \pm 0.2$ & $10.8 \pm 0.4$ \\
\hline
\end{tabular}

in Fig. 8, which is similar to Fig. 7 in the quality of the fit to the data.

The estimated HPLC is $(166 \pm 1) \mathrm{WK}^{-1}$ and HTC is $(152 \pm 1) \mathrm{WK}^{-1}$, which are both slightly higher than those from the analysis of the February data for this case study. Such differences could be caused by the ability of the models to describe seasonally-dependent variations in the recorded data, or by changes in occupant behaviour, such as the ventilation strategy. The effective thermal mass estimates are also higher than the
February data, with higher effective solar apertures reflecting that no other solar gain is included for February.

There are some features of the model results common to all periods of data analysed here, and can thus be considered to reflect on the model performance in general. Whilst providing a generally good fit to the data, as shown above, the residuals for all of the temperature predictions have clear structure indicating that some of the dynamic heat transfers processes within the dwellings have not been fully characterised by the presented models. The model predictions capture the trend of the data well, but peaks and troughs are often under- or over-estimated. Although this is partly expected as consequence of the thermal mass effect (which effectively acts as a low-pass filter in the equivalent electrical circuit), it also suggests that the models may be omitting or oversimplifying some of the thermophysical mechanisms occurring in the dwellings. Examples of these could be ventilation heat transfer, which may be particularly important for occupied homes with summer data, and metabolic gains. Averaging the effective solar apertures over each façade could also cause disparities in the tem- 


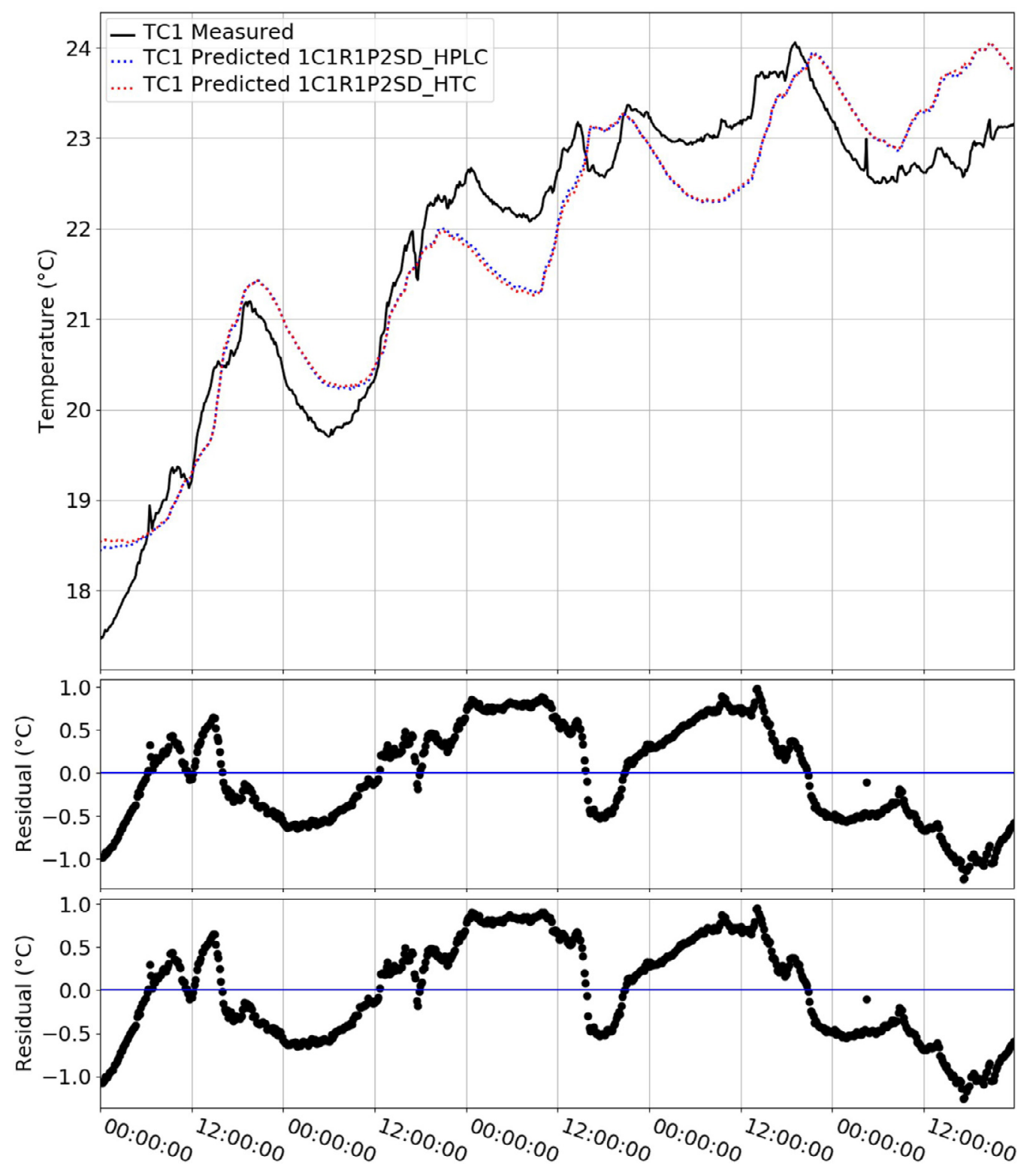

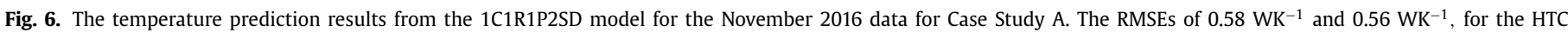
and HPLC plots respectively, are considerably larger than those for the prediction of the June 2017 data. The residuals are again in the order of the legend.

Table 4

The results for the HPLC and the HTC from all models outlined in Section 2.1.1 for both time periods in both case studies. The effect of the inclusion of solar gains is clearly shown, as is the effect of the season on the error. Results indicated in bold are those from the model with the best evidence for that data.

\begin{tabular}{|c|c|c|c|c|}
\hline \multirow[b]{2}{*}{ Model } & \multicolumn{4}{|l|}{$\operatorname{HPLC}\left(\mathrm{WK}^{-1}\right)$} \\
\hline & A, November & A, June & B, February & B, April \\
\hline 1C1R1P & $186 \pm 1$ & $61 \pm 2$ & $146.5 \pm 0.1$ & $109.3 \pm 0.2$ \\
\hline 1C1R1P1S & $183 \pm 2$ & $170 \pm 10$ & $167.3 \pm 0.3$ & $162 \pm 1$ \\
\hline 1C1R1PmS & $138 \pm 8$ & $190 \pm 10$ & $167.9 \pm 0.3$ & $166 \pm 1$ \\
\hline 1C1R1P(m-1)S & $137 \pm 7$ & $190 \pm 10$ & $167.9 \pm 0.3$ & $166 \pm 1$ \\
\hline 1C1R1PmSD & $172 \pm 6$ & $190 \pm 20$ & $137.4 \pm 0.9$ & $138.6 \pm 0.8$ \\
\hline 1C1R1P(m-1)SD & $163 \pm 5$ & $\begin{aligned} & 180 \pm 20 \\
& \text { HTC }\end{aligned}$ & $\begin{array}{r}137.4 \\
\left(W^{-1}\right)\end{array}$ & $138.6 \pm 0.8$ \\
\hline 1C1R1P & $169 \pm 1$ & $57 \pm 2$ & $134.7 \pm 0.1$ & $101.0 \pm 0.2$ \\
\hline 1C1R1P1S & $165 \pm 2$ & $160 \pm 10$ & $153.4 \pm 0.3$ & $149 \pm 1$ \\
\hline 1C1R1PmS & $117 \pm 8$ & $170 \pm 10$ & $153.9 \pm 0.3$ & $152 \pm 1$ \\
\hline 1C1R1P(m-1)S & $118 \pm 8$ & $170 \pm 10$ & $153.9 \pm 0.3$ & $152 \pm 1$ \\
\hline 1C1R1PmSD & $154 \pm 6$ & $170 \pm 20$ & $125.6 \pm 0.9$ & $127.7 \pm 0.7$ \\
\hline 1C1R1P(m-1)SD & $145 \pm 6$ & $170 \pm 20$ & $125.6 \pm 0.9$ & $127.7 \pm 0.7$ \\
\hline Coheating test result & 141 & 141 & 133 & 133 \\
\hline
\end{tabular}




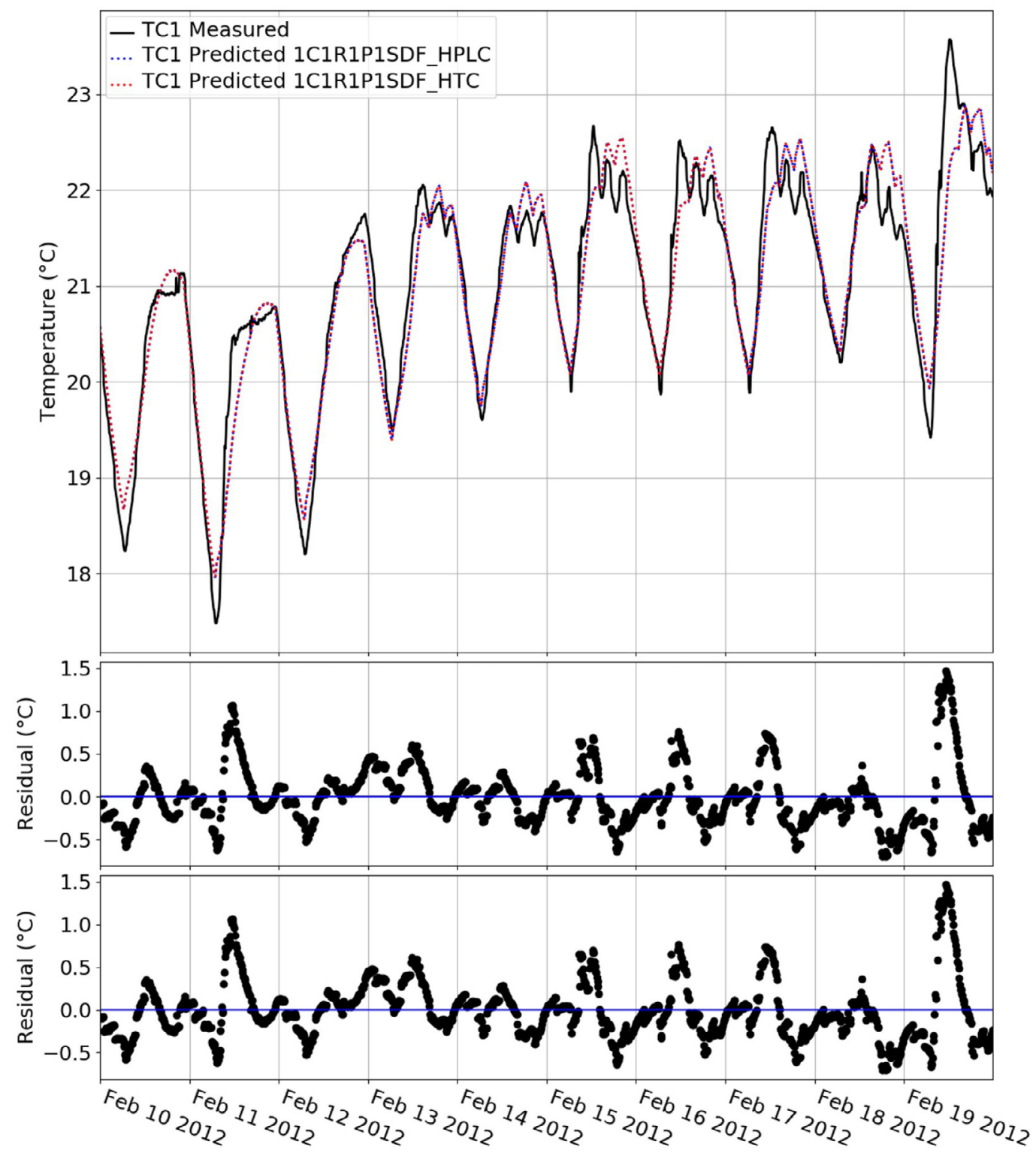

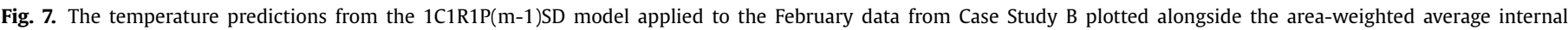
temperature measured during this period. The RMSE for these temperature predictions is $0.34 \mathrm{WK}^{-1}$ for both plots.

perature predictions compared to observed data, as this could decrease the modelled heat input from solar radiation at the internal temperature peaks. Another potential cause of the correlated residuals is that the effective thermal mass may not be at the same location as the area-weighted average internal temperature, as modelled here, or that multiple thermal masses are required to capture the complex behaviour of real dwellings. Finally, data was not available to this study that included typical water heating and usage patterns; further work is required to investigate and develop appropriate models to describe its impact on whole dwelling performance estimates.

Whilst the models presented in this paper are highly simplified, particularly in their treatment of the thermal mass, the general behaviour of the data is well represented in the predictions they produce. The models produce HTC estimates that are consistent over different seasons, given the expected differences due to the changing conditions, and any disparities between models are as would be expected given their differing parameters. This is expanded on in Section 3.2 .

\subsubsection{The importance of a model selection process}

The same model $(1 \mathrm{C} 1 \mathrm{R} 1 \mathrm{P}(\mathrm{m}-1) \mathrm{SD})$ was selected for the cooler data for both case studies, with the $1 \mathrm{C} 1 \mathrm{R} 1 \mathrm{P}(\mathrm{m}-1) \mathrm{S}$ model best able to describe the data from warmer months. The excluded façade was the same for both Case Study B periods, however differed for Case Study A. The two periods of data analysed for Case Study A span a wider seasonal difference than for Case Study B, which could be a factor in the selection of different models, as the relative contributions of different heat sources varies across the year. In particular, the size of the space heating load compared to the water heating load, shading and cloud cover may all change considerably for some dwellings. The results for both case studies also suggest that the HTC itself is not necessarily a constant quantity (as discussed above in Section 2.2.3), as expected, due to variations in weather affecting heat flow (particularly ventilation rates) and because occupant use of a property is likely to vary through the year, particularly with respect to window opening.

For all data analysed, model selection indicated that including $(\mathrm{m}-1)$ façades in the model best represented the data, with the 

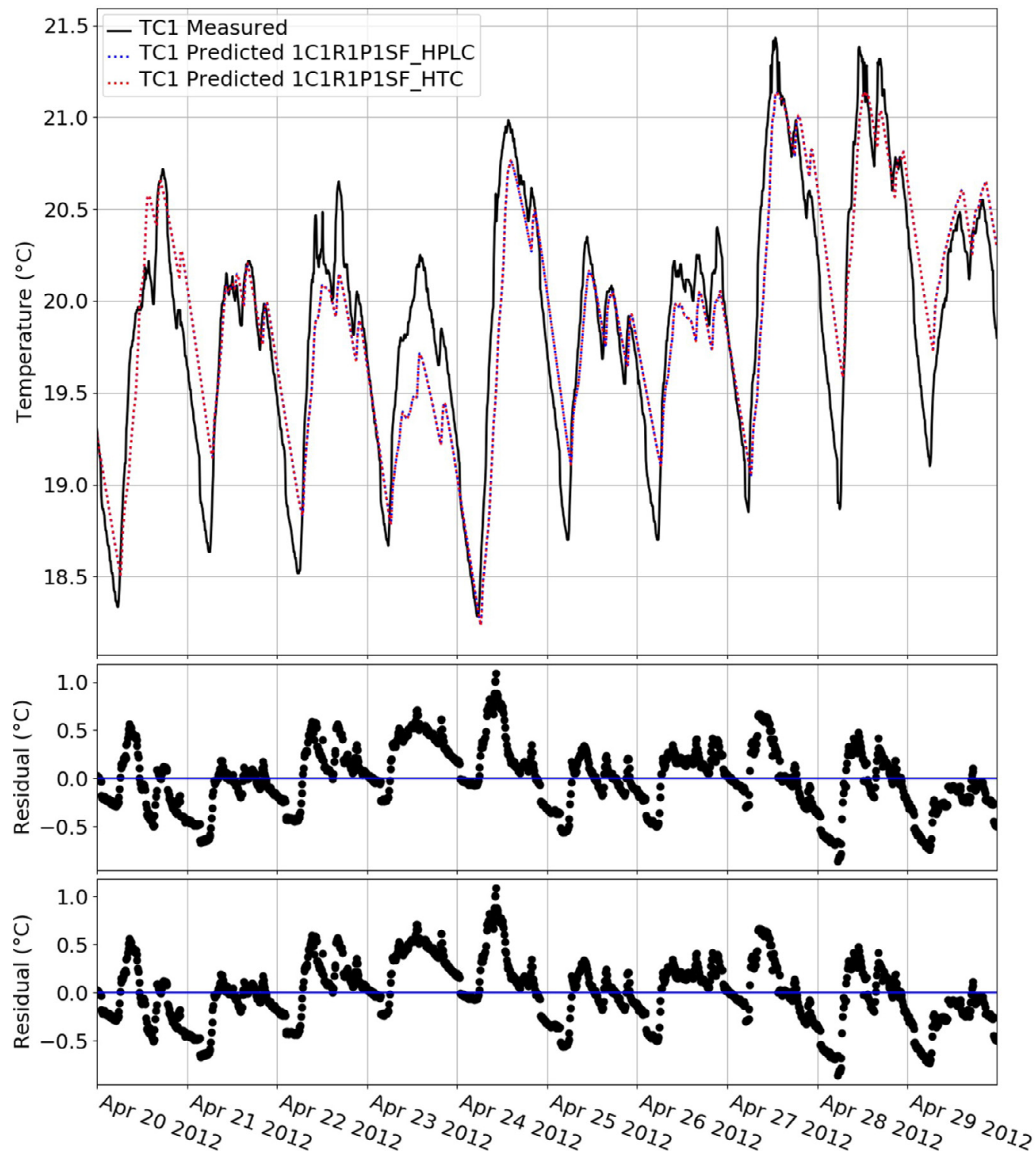

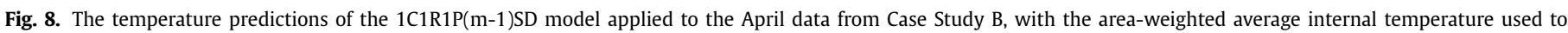

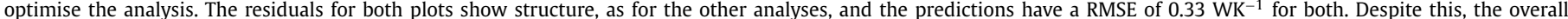
fit to the data appears reasonable.

NW façade of Case Study A excluded in June, and the NE façade in November. For Case Study B the NW façade was excluded for both periods of data analysed. Such differences in the models best able to represent the observed data across seasons highlights the advantages of the model selection process: differences in dwelling performance may be related to physical effects, and multiple models may be applied that represent different thermal characteristics and local conditions, particularly shading.

\subsection{Comparison of all results}

In Table 4 the means of the posterior distributions of the HTC and HPLC are shown for the models described in Section 2.1.1, with the estimates from the best model highlighted in bold, for each time period analysed. The means are used to summarise the distributions as they were found to be approximately Gaussian in all cases. As outlined in Section 2.2.3, the errors given in Table 4 are the standard deviations of the posterior distributions of the HTCs and HPLCs, and thus reflect only the confidence in the position of the mean given the model in question rather than estimating the full uncertainty. These are predominantly smaller for the HTCs and HPLCs estimated with winter data compared with the warmer data for the corresponding case studies, reflecting that there is less variation in input data (for instance, the internal-external temperature difference and gas use) during summer thereby providing less information to the model.

The errors in Table 4 are lower for Case Study B than Case Study A. The models presented in this paper assume that the dwelling is in a constant configuration during the data analysed, for example with all internal and external windows and doors remaining either open or closed throughout. In Case Study B this is likely to be accurate, as the building was unoccupied. However, Case Study A was occupied and such components of the building configuration thus increase the spread of the posterior parameter distributions.

Table 4 reports both the results of the coheating test and the dynamic analysis developed in this work. It is notable that considerably shorter datasets collected in-use dwellings were sufficient for the dynamic models: typically 5-10 days, compared to a stan- 
dard monitoring campaign of 2-3 weeks from unoccupied properties for coheating [18]. Similarly, the dynamic method enabled the estimation of the HTC and HPLC at times of year when coheating tests are not advised [18]: outside the heating season, including in the summer, with $<15 \%$ difference between the models with the highest evidence. A rapid and robust method for the characterisation of the thermophysical performance of occupied buildings at different times of the year has undoubtedly several advantages and practical applications. These include the use of the method as a tool for operational energy performance evaluation, accounting for the building's state of conservation and surrounding landscape; for diagnosis and quality assessment; for decision making and regulatory purposes.

The difference between HTC and HPLC estimates for different models incorporating solar gains is case-study specific and can highlight the characteristics of the property. The results display several characteristics that align to expectations. Firstly, all the results from the lumped capacitance models (including solar gains) are higher than the coheating test HTC estimates. This is to be expected as during coheating testing ventilation pathways are closed [18], whereas under normal operation they may be open, adding to the heat load. This effect is exacerbated by the fans used in coheating tests to ensure uniform air temperature throughout a building. For Case Study A the property was occupied during the analysed periods and therefore additional heat loss paths may be present, in addition to any occupant-driven effects on the energy consumption of the building.

The lowest HTC and HPLC for each case study and time period is mostly produced by the 1C1R1P model without solar gains. In this model the only heat supply to raise internal temperatures above external temperatures is assumed to be gas and electricity consumption, whilst in reality the unmetered solar gains also increase internal temperatures, resulting in an estimate of the HTC and HPLC that is too low. The difference between HTC and HPLC estimates for the 1C1R1P model and the others in the June and April results is pronounced, as expected due to the increased solar gains in these warmer months compared to winter.

For all data periods, the HTC and HPLC results from the solar models with separated inputs and diffuse radiation are in strong agreement between the $m$ and $(m-1)$ versions. This is because the models tend towards the same solution: the effective aperture of the façade in the model variant which is excluded for $(m-1)$ has been found to be approximately zero, and thus has little effect on the model results. This enables the plots of the MCMC traces, posterior parameter distributions, and covariances to be used to select the most appropriate model for a particular set of data (Section 3.1); in this case the Bayesian model selection between $\mathrm{m}$ and m-1 models does not improve the physical insights from their application but confirms the most appropriate model to describe the observed data.

The effect on the HTC and HPLC of the separate inclusion of diffuse radiation varies between the data analysed. Similarly to the inclusion of direct radiation, it is likely that failing to account for diffuse radiation into a dwelling neglects a potentially significant heat source, which lowers the HTC and HPLC estimated by such models; the importance of this factor changes seasonally as demonstrated by the differences in the models with the highest evidences between different data.

In Table 4 both the HTC and HPLC are given, with the former using the metered gas consumption multiplied by the manufacturer boiler efficiency, and the latter the metered gas consumption alone. As would be expected, the HTC results are lower than the corresponding HPLCs in all cases, as the HPLC includes losses from the heating systems, whilst the HTC excludes them. The HTCs are directly comparable to the coheating test results; however, as the real efficiency of the boiler and how it changes with season is un- known [51-53], using this fixed efficiency introduces an important assumption and potential cause of error into the analysis. The alternative HPLC represents the fabric efficiency in addition to the heating plant efficiency and, whilst not simply comparable to the HTC, represents the overall thermal performance of the dwelling in its measured and operational state.

\section{Conclusions}

In this paper a series of lumped thermal capacitance models of in-use whole-house thermal performance have been presented. These models have incorporated solar gains in different ways; their performance has been analysed with respect to data from two case studies using Bayesian techniques with MCMC sampling. It has been illustrated that heat input from solar radiation is crucial for the evaluation of whole-building in-situ thermal performance at all times of the year, and also that this can be estimated in summer and winter using datasets of 5-10 days in an occupied dwelling, in contrast to standard steady-state methods and co-heating requiring a number of weeks. The models that best describe the data, as determined by Bayesian model comparison, are seasonally and building dependent. HTCs at different times of the year were within $15 \%$ of each other, including during the summer, and in line with coheating results.

The models presented in this paper all include one thermal mass, one thermal resistance and heat input from electrical gains and the heating system. Models have been applied that incorporate direct solar radiation as a single heat gain over the whole fabric, as a gain to each façade separately, as a gain to a reduced number of façades, and to the latter two also including diffuse radiation. The practical and structural (both local and global) identifiability was assessed for these models. The global structural identifiability was investigated utilising the transfer function approach in the Laplace domain under mild assumptions about the input data; investigations of conditions or combinations of inputs breaking these assumptions would be interesting for future work. The ability of each model to explain the observed case study data was tested using Bayesian model comparison, reviewing the MCMC chains and covariance plots, and the RMSE.

The model that best explains observed data is dependent on both the building and the time of year, with different façades more significant in the different seasons; separated façades were required for both seasons' data. It is notable that in both of the case studies presented in this paper, models that exclude one of the exposed façades performed best in Bayesian model comparison. This is likely to be highly case specific, depending on the glazed areas and the potentially seasonally dependent local shading that they experience, as demonstrated by the results for Case Study A. The derived models can therefore estimate the relative significance of solar gains, façade-by-façade at different times of year, providing insight into the dwelling's dynamic performance.

Models separately including direct and diffuse radiation were selected as the most appropriate for two of the datasets analysed in this paper, demonstrating that different treatment of these aspects of solar radiation is often necessary. The periods of data where separate accounting of direct and diffuse radiation was not found to be necessary were in warmer months and have higher levels of solar radiation: direct radiation dominated during these times, highlighting the dependence of the model that best describes the thermal performance of a property on the weather.

The estimates of a dwelling's HTC can vary considerably between the different models and at different times of year; most notably for the data from the warmer months of June (Case Study A) and April (Case Study B). In both of these instances, the result for the 1C1R1P model is significantly lower than those from all other models, and indeed also that from the coheating test. Such results 
are expected, due to the impact of the unmetered solar gains on internal temperatures, and highlight the importance of accounting for solar radiation in such analysis. By contrast, there is little difference in the HTC estimates for the $m$ and $m-1$ versions of the models selected as best in each case, as expected when the additional aperture is approximately zero.

An enhanced method, like the one presented here, enabling the rapid and robust evaluation of heat transfer and thermophysical behaviour of occupied dwellings at any time of the year, has the potential to reduce the performance gap by extending the use of monitored data to gain greater understanding of the energy performance of the building stock. Methods, such as that presented here, that can be used to automatically select the best physical representation of a dwelling through the specific model that is applied, can highlight how the building performance changes over the course of a year, and identify models that do not represent the data well. The ability to apply such methods in the future is likely to increase, due to the availability of data from smart meters, extensive weather data, internet of things sensors and smart building controls [58]. With further development of the method and the models, such techniques may be used to provide tailored retrofitting solutions; to inform occupants of appropriate space heating and cooling strategies to improve thermal comfort; to evaluate the thermal performance of dwellings to assess building compliance and provide feedback loops to the building industry; and to provide new business and policy opportunities in the energy sector towards closing the performance gap.

\section{Author Declaration}

Frances Hollick Conceptualisation, Methodology, Software, Formal analysis, Investigation, Data Curation, Writing - Original Draft, Visualisation, Project administration Virginia Gori Methodology, Software, Validation, Writing - Review \& Editing, Funding acquisition Cliff Elwell Conceptualisation, Writing - Review \& Editing, Supervision, Funding acquisition

\section{Declaration of Competing Interest}

We wish to confirm that there are no known conflicts of interest associated with this publication and there has been no significant financial support for this work that could have influenced its outcome.

\section{Acknowledgments}

This research was made possible by support from the EPSRC Centre for Doctoral Training in Energy Demand (LoLo), grant numbers EP/L01517X/1 and EP/H009612/1, and the sponsorship of Wilmott Dixon. It was also supported by the Research Councils UK (RCUK) Centre for Energy Epidemiology, EP/K011839/1, the EPSRC Centre for Research into Energy Demand Solutions, EP/R035288/1, and the EPSRC Doctoral Prize fellowship, EP/N509577/1. The authors are grateful to Dr Jez Wingfield for assistance and advice concerning the monitoring campaign for Case Study A, and to Dr Phillip Biddulph for the original development of a MAP-based Bayesian approach. They are also grateful to the team at Leeds Beckett for providing the dataset for Case Study B.

\section{Appendix A. Investigation of global structural identifiability}

Global structural identifiability (GSI) was investigated for the lumped thermal capacitance models presented in Section 2.1.1, under the input datastreams combination covered in this paper (i.e. non-zero power and solar inputs, and non-constant non-zero temperature inputs). The transfer function method [33] in the Laplace domain was adopted to assess GSI. This method is particularly suited to the framework presented, as the Laplace transform is already used as intermediate step to replace the linear differential equations with polynomial operations in the complex variable $\mathbb{S}$ (representing the derivative operator) before discretising the models for simulations $[30,39]$.

Single thermal mass model with power input (1C1R1P) Using the Laplace transform, the governing differential equation for the 1C1R1P model (Eq. (1), Section 2.1.1) can be rewritten as:

$C_{1} \mathbb{S} T_{\text {in }}(\mathbb{S})=\frac{T_{\text {out }}(\mathbb{S})-T_{\text {in }}(\mathbb{S})}{R_{1}}+P(\mathbb{S})$

Dividing Eq. (A.1) by $C_{1}$ and rearranging:

$\left(\mathbb{S}+\frac{1}{R_{1} C_{1}}\right) T_{\text {in }}(\mathbb{S})=\frac{T_{\text {out }}(\mathbb{S})}{R_{1} C_{1}}+\frac{P(\mathbb{S})}{C_{1}}$,

it can be observed that for arbitrary sets of parameters $\theta$ and $\theta^{\prime}: R_{1} C_{1}=R_{1}^{\prime} C_{1}^{\prime}, C_{1}=C_{1}^{\prime}$ and therefore $R_{1}=R_{1}^{\prime}$, demonstrating that the 1C1R1P model is globally structurally identifiable for the input datastreams combination relevant to this paper. Interestingly, there are pathological cases where the model may become nonidentifiable. For example, when the power is constantly zero the last term in Eq. (A.2) vanishes and the model is non-identifiable along hyperboles $R_{1} C_{1}=$ const.

Single thermal mass model with power and solar input (1C1R1P1S) Following the same approach adopted above for the 1C1R1P model, the governing differential equation for the 1C1R1P1S model (Eq. (2), Section 2.1.1) can be rewritten as:

$C_{1} \mathbb{S} T_{\text {in }}(\mathbb{S})=\frac{T_{\text {out }}(\mathbb{S})-T_{\text {in }}(\mathbb{S})}{R_{1}}+P(\mathbb{S})+g S(\mathbb{S})$.

Dividing Eq. (A.3) by $C_{1}$ and rearranging:

$\left(\mathbb{S}+\frac{1}{R_{1} C_{1}}\right) T_{\text {in }}(\mathbb{S})=\frac{T_{\text {out }}(\mathbb{S})}{R_{1} C_{1}}+\frac{P(\mathbb{S})}{C_{1}}+\frac{g S(\mathbb{S})}{C_{1}}$.

it can be observed that for arbitrary sets of parameters $\theta$ and $\theta^{\prime}$ : $R_{1} C_{1}=R_{1}^{\prime} C_{1}^{\prime}, C_{1}=C_{1}^{\prime}, g=g^{\prime}$ and therefore $R_{1}=R_{1}^{\prime}$, demonstrating that also the 1C1R1P1S model is globally structurally identifiable under the input conditions relevant to this paper.

Following the same reasoning as above and under similar assumptions about the data, it can be demonstrated the GSI of the remaining two models presented in Section 2.1.1: the 'Single thermal mass model with power and separate solar input per facade' (1C1R1PmS) and the 'Single thermal mass model with power, direct solar input per facade and diffuse solar input' (1C1R1PmSD).

\section{References}

[1] P. Nejat, F. Jomehzadeh, M.M. Taheri, M. Gohari, M.Z. Muhd, A global review of energy consumption, $\mathrm{CO} 2$ emissions and policy in the residential sector (with an overview of the top ten $\mathrm{CO} 2$ emitting countries), Renew. Sustain. Energy Rev. 43 (2015) 843-862, doi:10.1016/j.rser.2014.11.066.

[2] OECD, Transition to sustainable buildings: Strategies and opportunities to 2050, 2013, doi:10.1787/9789264202955-en.

[3] Zero Carbon Hub, Closing the gap between Design and as-built performance, Evidence Review Report, 2014. March

[4] D. Johnston, D. Miles-Shenton, D. Farmer, Quantifying the domestic building fabric 'performance gap', Build. Serv. Eng. Res.Technol. 36 (5) (2015) 614-627, doi: $10.1177 / 0143624415570344$.

[5] J. Wingfield, M. Bell, D. Miles-Shenton, T. South, B. Lowe, Lessons from Stamford Brook. Understanding the Gap between Designed and Real Performance.(2009) 1-137.

[6] M. Sunikka-Blank, R. Galvin, Introducing the prebound effect: the gap between performance and actual energy consumption, Build. Res. Inf. 40 (3) (2012) 260-273, doi:10.1080/09613218.2012.690952.

[7] BS ISO 52016-1, BS ISO 52016-1:2017. Energy performance of buildings - Energy needs for heating and cooling, internal temperatures and sensible and latent heat loads. (2017).

[8] M.F. Fels, PRISM : an introduction, Energy Build. 9 (1986) 5-18.

[9] J. Chapman, R. Lowe, R. Everett, The Pennyland Project (1985).

[10] A.J. Summerfield, R.J. Lowe, T. Oreszczyn, Two models for benchmarking UK domestic delivered energy, Build. Res. Inf. 38 (1) (2010) 12-24, doi:10.1080/ 09613210903399025. 
[11] A.J. Summerfield, T. Oreszczyn, I.G. Hamilton, D. Shipworth, G.M. Huebner, R.J. Lowe, P. Ruyssevelt, Empirical variation in 24-h profiles of delivered power for a sample of UK dwellings: implications for evaluating energy savings, Energy Build. 88 (2015) 193-202, doi:10.1016/j.enbuild.2014.11.075.

[12] S. Danov, J. Carbonell, J. Cipriano, J. Martí-Herrero, Approaches to evaluate building energy performance from daily consumption data considering dynamic and solar gain effects, Energy Build. 57 (2013) 110-118, doi:10.1016/j. enbuild.2012.10.050.

[13] A. Rabl, A. Rialhe, Energy signature models for commercial buildings: test with measured data and interpretation, Energy Build. 19 (2) (1992) 143-154, doi:10. 1016/0378-7788(92)90008-5.

[14] I. Knight, G. Dunn, R. Gaddas, Improved methods for evaluating base temperature for use in building energy performance lines, Build. Serv. Eng. Res. Technol. 4 (2003) 221-228.

[15] C. Ghiaus, Experimental estimation of building energy performance by robust regression, Energy Build. 38 (6) (2006) 582-587, doi:10.1016/j.enbuild.2005.08. 014.

[16] A. Caucheteux, M. Nedelec, U. Bramkamp, A Building Energy Efficiency CHAracterisation Method (BEECHAM) to Assess Existing Buildings Performance, in: 14th International Conference on Computing in Civil and Building Engineering, 2012, pp. 27-29. Moscow

[17] J. Berger, S. Tasca-Guernouti, M. Humbert, Experimental method to determine the energy envelope performance of buildings, in: Proceedings of the Tenth International Conference for Enhanced Building Operations, Kuwait, October, 2010.

[18] J. Wingfield, D. Johnston, D. Miles-Shenton, M. Bell, Whole House Heat Loss Test Method (Coheating), 2010,

[19] R. Jack, Building Diagnostics : Practical Measurement of the Fabric Thermal Performance of Houses, Loughborough, 2015 Ph.D. thesis.

[20] J. Palmer, G. Pane, J. Wingfield, M. Bell, Comparing Primary and Secondary Terms Analysis and Re-Normalisation (PSTAR) Test and Co-Heating Test Results, Technical Report, 2011.

[21] P. Boisson, R. Bouchie, ISABELLE method: In-situ assessment of the building envelope performances, in: 9th International Conference on System Simulation in Buildings, 2014, pp. 1-20.

[22] A. Brun, P. Boisson, S. Thebault, Short methodologies for in-situ assessment of the intrisinc thermal performance of the building envelope, Sustain. Places (October) (2014).

[23] F.P. Incropera, Fundamentals of Heat and Mass Transfer., 6th ed., John Wiley, 2007.

[24] E. Mangematin, G. Pandraud, D. Roux, Quick measurements of energy efficiency of buildings, Comptes Rendus Physique 13 (4) (2012) 383-390, doi:10. 1016/j.crhy.2012.04.001.

[25] Crabb, A simplified thermal response model, Build. Serv. Eng. Res. Tecnol. 8 (1987) (1987) 13-19.

[26] K.K.D. Andersen, H.D. Madsen, L.H.R. Hansen, Modelling the heat dynamics of a building using stochastic differential equations, Energy Build. 31 (1) (2000) 13-24, doi:10.1016/S0378-7788(98)00069-3.

[27] S. Wang, X. Xu, Parameter estimation of internal thermal mass of building dynamic models using genetic algorithm, Energy Convers. Manage. 47 (13-14) (2006) 1927-1941, doi:10.1016/j.enconman.2005.09.011.

[28] X. Xu, S. Wang, A simplified dynamic model for existing buildings using CTF and thermal network models, Int. J. Therm. Sci. 47 (9) (2008) 1249-1262, doi:10.1016/j.ijthermalsci.2007.10.011.

[29] Q. Zhou, S. Wang, X. Xu, F. Xiao, A grey-box model of next-day building thermal load prediction for energy-efficient control, Int. J. Energy Res. 32 (15) (2008) 1418-1431, doi:10.1002/er.1458.

[30] V. Gori, P. Biddulph, C.A. Elwell, A Bayesian dynamic method to estimate the thermophysical properties of building elements in all seasons, orientations and with reduced error, Energies 11 (4) (2018) 802, doi:10.3390/en11040802.

[31] J.F. Orgill, K.G.T. Hollands, Correlation equation for hourly diffuse radiation on a horizontal surface, Solar Energy 19 (4) (1977) 357-359, doi:10.1016/ 0038-092X(77)90006-8.

[32] J.A. Duffie, W.A. Beckman, Solar Engineering of Thermal Processes Solar Engineering, 2013, doi:10.1002/9781118671603.fmatter.

[33] E. Walter, L. Pronzato, Qualitative and quantitative experiment design for phenomenological models-A survey, Automatica 26 (2) (1990) 195-213, doi:10. 1016/0005-1098(90)90116-Y.
[34] P. Gregory, Bayesian Logical Data Analysis for the Physical Sciences: a Comparative Approach with "Mathematica" Support, 2005, doi:10.1017/ CBO9780511791277.

[35] D. Foreman-Mackey, D.W. Hogg, D. Lang, J. Goodman, Emcee : the MCMC hammer, Publications Astronom. Soc.Pac. 125 (925) (2013) 306-312, doi:10.1086/ 670067.

[36] J. Goodman, J. Weare, Ensemble samplers with affine invariance, Commun Appl. Math.Comput. Sci. 5 (2) (2010).

[37] S. Rouchier, Solving inverse problems in building physics: an overview of guidelines for a careful and optimal use of data, Energy Build. 166 (2018) 178195, doi:10.1016/j.enbuild.2018.02.009.

[38] V. Gori, C.A. Elwell, Estimation of thermophysical properties from in-situ measurements in all seasons: quantifying and reducing errors using dynamic greybox methods, Energy Build. 167 (2018) 290-300, doi:10.1016/j.enbuild.2018.02. 048.

[39] V. Gori, V. Marincioni, P. Biddulph, C.A. Elwell, Inferring the thermal resistance and effective thermal mass distribution of a wall from in situ measurement to characterise heat transfer at both the interior and exterior surfaces, Energy Build. 135 (2016) 398-409, doi:10.1016/j.enbuild.2016.10.043.

[40] V. Gori, A Novel Method for the Estimation of Thermophysical Properties of Walls from Short and Seasonally Independent In- Situ Surveys, University College London, 2017 Ph.D. thesis.

[41] F. Hollick and J. Wingfield, Dataset: Monitoring campaign of an occupied UK dwelling, 2019. doi:10.14324/000.ds.10087216.

[42] R. Jack, Low Energy Retrofit Performance Measurement Report, Technical Report, 2016.

43] Eltek Ltd., GenII Transmitters for humidity and temperature, 2019a,

[44] Eltek Ltd., GenII Transmitter type GD47, 2019b,

[45] Eltek Ltd., Energy Monitoring - Pulse, 2019c,

[46] Eltek Ltd., GenII External RH and Temperature Transmitter Type OD13J and OD14J, 2019d,

[47] Kipp \& Zonen, Pyranometers For the Accurate Measurement of Solar Irradiance, 2016,

48] D. Miles-Shenton, J. Wingfield, R. Sutton, M. Bell, Temple Avenue field tria Evaluation of design \& construction process and measurement of fabric performance of new build dwellings, Technical Report, Leeds Beckett University, 2010.

[49] Orsis UK, Orsis (UK) Leading the way in Remote Metering, 2019,

[50] SAP, SAP2012 - The Government' s Standard Assessment Proceedure for Energy Rating of Dwellings (October 2013) (2012).

[51] G. Bennett, C. Elwell, R. Lowe, T. Oreszczyn, The importance of heating system transient response in domestic energy labelling, Buildings 6 (3) (2016) 29, doi:10.3390/buildings6030029.

[52] G. Bennett, C. Elwell, T. Oreszczyn, Space heating operation of combination boilers in the UK: the case for addressing real-world boiler performance, Build Serv. Eng. Res.Technol. 40 (1) (2019) 75-92, doi:10.1177/0143624418794552.

[53] G. Orr, T. Lelyveld, S. Burton, In-situ monitoring of efficiencies of condensing boilers and use of secondary heating, DECC Energy Saving Trust 1 (June) (2009) 44

[54] J.D. Chambers, T. Oreszczyn, Deconstruct: a scalable method of as-built heat power loss coefficient inference for UK dwellings using smart meter data, Energy Build. 183 (2019) 443-453, doi:10.1016/j.enbuild.2018.11.016.

[55] A. Gelman, Prior Choice Recommendations, 2019, (https://github.com/ stan-dev/stan/wiki/Prior-Choice-Recommendations)[Online; accessed 07Aug-2019].

[56] The Scipy Community, scipy.optimize.basinhopping, 2016, (https://docs. scipy.org/doc/scipy-0.17.1/reference/generated/scipy.optimize.basinhopping. html)[Online; accessed 07-Aug-2019].

[57] BS ISO 9869-1, BS ISO 9869-1:2014 Thermal insulation Building elements In-situ measurement of thermal resistance and thermal transmittance Part 1 Heat flow meter method, Technical Report, 2014.

[58] I. Khajenasiri, A. Estebsari, M. Verhelst, G. Gielen, A review on internet of things solutions for intelligent energy control in buildings for smart city applications, Energy Procedia 111 (September 2016) (2017) 770-779, doi:10.1016/ j.egypro.2017.03.239. 